\title{
Unified Driver Model simulation and its application to the automotive, rail and maritime domains
}

\author{
Pietro Carlo Cacciabue, Simon Enjalbert, Hakan Soderberg and Andreas Tapani
}

\section{Linköping University Post Print}

\section{Tweet}

N.B.: When citing this work, cite the original article.

Original Publication:

Pietro Carlo Cacciabue, Simon Enjalbert, Hakan Soderberg and Andreas Tapani, Unified Driver Model simulation and its application to the automotive, rail and maritime domains, 2013, Transportation Research Part F: Traffic Psychology and Behaviour, (21), 315-327. http://dx.doi.org/10.1016/j.trf.2013.09.020

Copyright: Elsevier http://www.elsevier.com/

Postprint available at: Linköping University Electronic Press http://urn.kb.se/resolve?urn=urn:nbn:se:liu:diva-104133 


\title{
Unified Driver Model simulation and its application to the automotive, rail and maritime domains
}

\author{
P. Carlo Cacciabue ${ }^{1 *}$ \\ ${ }^{1}$ KITE Solutions, srl, \\ V. Labiena 93, \\ 21014 Laveno \\ Mombello (VA) \\ Italy
}

${ }^{*}$ Corresponding Author

\author{
Simon Enjalbert ${ }^{2}$, \\ ${ }^{2}$ Univ Lille Nord de \\ France, \\ UVHC, LAMIH, \\ CNRS, UMR 8201, \\ F-59313 Valenciennes \\ France
}

\author{
Håkan Söderberg ${ }^{3}$, \\ ${ }^{3}$ Department of Shipping \\ and Marine Technology, \\ Chalmers University of \\ Technology, \\ Goteborg \\ Sweden
}

\author{
Andreas Tapani ${ }^{4}$ \\ ${ }^{4}$ Swedish National \\ Road and Transport \\ Research Institute \\ (VTI) and Department \\ of Science and \\ Technology, Linköping \\ University, Linköping, \\ Sweden
}

\begin{abstract}
This paper describes the implementation of a model of a driver into a computerised numerical simulation. The model is developed to capture the essential characteristics and common aspects of cognition and behaviour of a human being in control of a "vehicle" in different surface transport systems, namely trains, cars and ships. The main functions of the simulation are discussed as well as the experiments carried out in different types of driving simulators to support the estimation of the parameters utilised in the numerical simulation. The validation processes carried out in the rail and maritime domains are also discussed together with a critical review of capacities and limitations of the proposed approach.
\end{abstract}

\section{Introduction}

The main objective of this paper is to discuss and show how a Unified Model of Driver behaviour (UMD), developed to account for general and common aspect of cognition and behaviour for all surface transport modes, has been implemented in a numerical simulation architecture, and tuned and validated with respect to empirical evidence.

The UMD is based on the concept of the "joint" DVE (Driver-Vehicle-Environment) cognitive system, where the dynamic interactions between driver, vehicle and environment are represented in a harmonized and integrated manner. The model aims to represent the interaction between Driver-Vehicle-Environment in a simple way, which retains the essential correlations between some critical "independent variables" and enables prediction of driver behaviour in dynamic and rapidly changing conditions. In particular, the model focuses on a well-defined architecture of cognitive and behavioural performance of a driver (Carsten, 2007).

The overall Unified Model of Driver behaviour (UMD), described elsewhere (Oppenheim and Shinar, 2012), is based on the concept of parameters which enable the consideration of dynamic behaviour and interaction between the three components of the DVE system. Five parameters, namely attitude, experience, driver state, task demand and culture, are considered as the basic elements affecting behaviour.

From the simulation point of view, the correlations that link these parameters with observable variables are open, as different domains implies different actions that can be performed by the 
driver and, therefore, different dependencies between variables and components of the simulation. In essence, the implementation of the UMD in a numerical simulation requires taking in consideration different interfaces between the "driver" and the other constituents of the DVE system and the dynamic/continuous evaluation of the parameters on the basis of observable/calculated variables at each time interval of the DVE simulation. For instance, a car driver has to be able to steer, while this is not required by a train driver, even if they share the same behavioural model.

For these reasons different modules have been implemented, each one adapting the generic UMD to a different domain. Figure 1 shows how this choice has been accomplished in the simulation:

- The UMD has been implemented, accounting, at a higher level, for common aspects shared by all types of driver, including parameters representing mental status and the reciprocal correlations affecting their values.

- At a lower level, specific models of drivers, defining particular behaviour for distinct domains are provided, focusing on domain specific goals and tasks.

- The same strategy is adopted for the models of the vehicle and the environment.

In Figure 1 the full hierarchy of the models is shown, where dashed arrows represents the interactions between the three topmost level models (in red) and between the train related models (in green).

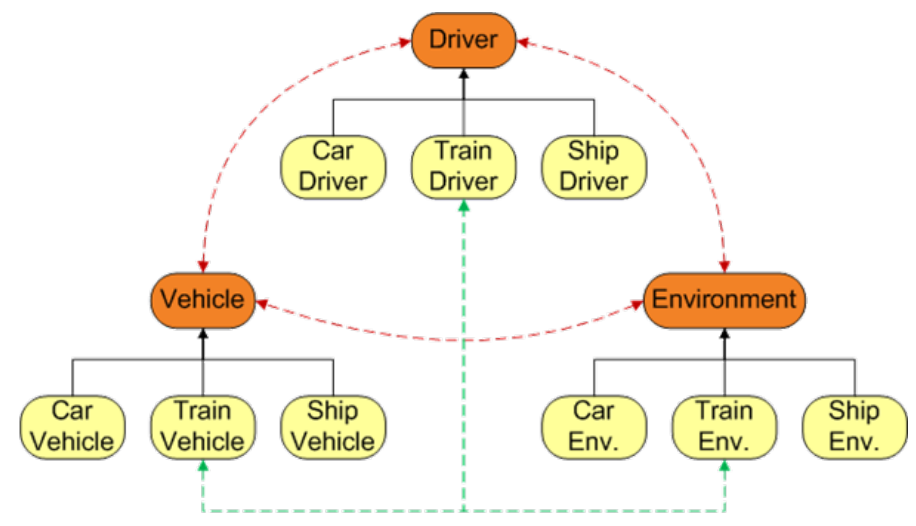

Figure 1. Simulation Architecture of the UMD in a DVE system interaction.

In this paper, the way in which the numerical simulation of the UMD has been developed will be discussed in detail. Then, the specific tuning process carried out for the automotive and rail domains will be presented. This process enabled the introduction of the above mentioned differences in driving tasks while retaining the overall architecture and modelling characteristics. The validation process with field experiments and the extension to the maritime domain will be subsequently discussed. Finally, achievements, future research and differences compared to other existing micro-simulation approaches will be discussed. 


\section{Simulation of the Unified Model of Driver Behaviour}

\subsection{UMD Simulation within an Overall DVE Architecture}

System modelling requires knowledge of theories and conservation principles, which must be combined with imagination and vision about ways to manage the resulting formulations and equations, in such a way that they are solvable and enable to predict dynamic behaviour, given certain boundaries and initial conditions. In most domains, e.g., nuclear physics, thermo hydraulics etc., the equations and correlations resulting from the theoretical modelling of systems and phenomena cannot be solved analytically and require simplifications of various nature by linearizing the dependencies between variables and coefficients, or by making strong assumptions about the overall behaviour of components.

Simulation development is the process by which a model is further reduced in terms of complexity by transforming the equations and correlations of the model into numerical algorithms, which are then implemented in a computer program, used to actually predict system dynamic behaviour.

Modelling and simulation are necessary in order to obtain a prediction approach that preserves the essential elements of the theory, while enabling to calculate system behaviour in a reasonable amount of time and computer power. In many cases, modelling and simulation are not two separate sequential processes, but a certain amount of iteration between them is necessary. This ensures that an adequate balance is kept between the need to capture the essential behavioural characteristics of a system and the need to calculate the system's dynamic evolution, given boundary and initial conditions, in a simple and fast running way.

There is a crucial step in the process of modelling and simulation that consists of the identification of the parameters and correlations which are most relevant and which enable combination of model variables into a description of the real behaviour of the system. As mentioned earlier, the parameters are quantities that enable to characterise the driver model, whereas the correlations are relationships between the parameters and some observable "independent variables" that characterise the human-machine interaction. These correlations are normally developed from an extensive number of experiments and field tests of various nature, as well as appropriate and accurate data analyses. The dynamic nature of the human-machine interaction generates time changes in the independent variables and, consequently, in the numerical value of the parameters, via the correlations, and, ultimately, it produces the dynamic changes in the simulation of human behaviour.

In the case of the UMD, the choice of the modeller has been to develop a theoretical formulation that is sufficiently simple and generic to capture the fundamental theories for each of the elements of the DVE system, especially with respect to the driver model. The simulation approach, called SiMUD (Simulation of Model of Universal Driver), aims at combining the simple nature of the UMD associated with the simulations of vehicle and environment models, within the overall DVE simulation architecture (Figure 2). In order to correlate the interplay of the three components of the DVE system, it is necessary that the parameters governing driver performance are calculated at each time interval of the simulation on the basis of the variables that are measured, or calculated, from the other modules and the driver model itself 


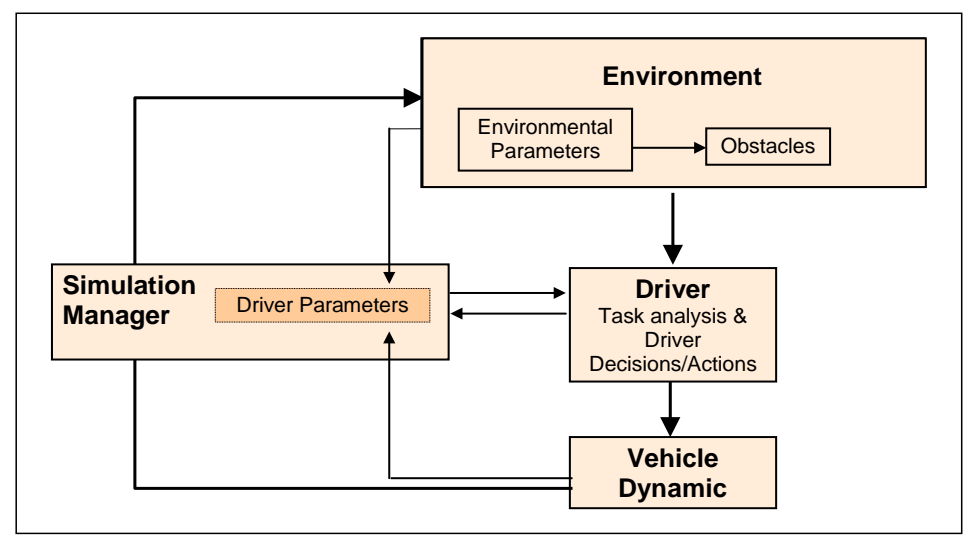

Figure 2. Overall Driver-Vehicle-Environment (DVE) system.

\subsection{SiMUD Dynamic Cognitive Functions and Processes}

\subsubsection{UMD Background and key Parameters}

In order to implement the UMD in a dynamic DVE simulation it is necessary that the cognitive functions and processes that generate the actions of the driver are considered. These are implemented in the simulation in a quite simple architecture that attempts to give a realistic description of the complexities of driver behaviour, i.e., a driver affected by personal attitudes, motivational aspects, workload, etc. Moreover, the overall requirement of the simulation is of being predictive and fast running, accounting also for interactions, human errors and adaptive behaviour.

The proposed approach for representing driver behaviour is to combine the well-known Information Processing System (IPS) paradigm (Neisser, 1967), with a simple Hierarchical Task Analysis (HTA) systematic method (Kirwan and Ainsworth, 1992). The level of accuracy of the analysis and the description of the tasks performed by the driver also define the granularity of the simulation (Michon, 1985). By means of HTA, the performance of a driver can be formalised and structured in a sequence of actions and goals that are carried out during the interaction with the vehicle and the environment.

In order to be a useful tool, the selected model should account for factors that have been shown to influence risk, risk-taking and errors. The selected driver parameters, chosen as input for the model and for the development of the simulation of the driver, are: Attitudes/personality, especially relevant for the automotive domain, Experience, Driver State, Task Demand and Culture. For a complete discussion on the model UMD and these parameters, please see Oppenheim and Shinar (2012). The parameters have been associated to specific factors that enable to assess their relative measure. The following associations have been made: Attitudes/personality has been measured by assessing the level of Sensation Seeking; Experience has been evaluated in terms of hazard perception skills; Driver State has been manipulated by induced fatigue from after lunch dip; Task Demand has been generated by subjective workload derived from a secondary task; and Culture has been associated to the country of origin of "drivers".

There are obvious differences between the different transport modes concerning these parameters, but they were deemed to be sufficient to give a reasonable cover of the majority of the important and relevant factors. 


\subsubsection{Decision Making, Task Performance and Implementation Mechanisms}

The decision making process and the execution of actions are the governing functions of SiMUD. A very simple formulation has been chosen, based on the driver's assessment of the minimum time to reach its destination. The vehicle is "driven" at the highest "intended" speed which is, in general, a function of the "maximum" allowed speed. This depends on the rules, regulations and contextual conditions, i.e., for the automotive environment, speed limits and traffic/road conditions. The meaning of maximum desired speed varies according to the application domain and can depend on driver parameters, speed limits, vehicle characteristics, time scheduling, etc.

In this simulation approach, rather than focusing on the detailed decision making process, a basic concept of similarity between different transportation modes has been developed.

In general, various driving modes can be simulated according to the choice and execution of different tasks. These vary from domain to domain, i.e., car, train or ship, and can be summarized, in a first instance, in the following 4 main tasks: Cruise; Overtake / Lane change; Follow; and Start / Stop. The execution of these tasks is represented by means of several variables and functions that account for driver personal aspects, vehicle characteristics and environmental conditions.

In order to capture the effects of the 5 basic parameters of the UMD that affect behaviour, i.e., Attitude, Experience, Driver State, Task Demand and Culture (ATT, EXP, DS, TD and CULT), the SiMUD implements the following assumptions:

1. The values of each parameter are implemented by means of discrete quantities.

2. These have been selected in such a way as to enable the association of essential variables of DVE interaction, e.g., speed, distance, acceleration and braking mode, with expected values.

3. The resulting formulations are then tuned according to the results of experimental observations, as discussed in next section.

4. This process and the use of experimental studies enable to differentiate between types of drivers as well as between cultures.

The following discrete values of the parameters have been associated to different types of driver and contextual conditions (Table I).

Table I. Basic parameters values characterising driver behaviour

\begin{tabular}{|cccc|}
\hline ATT & 0 & & $1 / 8$ \\
& (Prudent) & & (Sensation seeker) \\
EXP & $-1 / 8$ & & $1 / 8$ \\
& (Novice) & & (Experienced) \\
DS & $1 / 8$ & & $-1 / 8$ \\
& (Alert) & & (Fatigued) \\
TD & $1 / 8$ & 0 & $-1 / 8$ \\
& (Low) & (Medium) & (High) \\
\hline
\end{tabular}

CULT is considered separately and is not intended to have a numeric value, but is instead used to identify the nationality of the driver. 
The basic assumption of the simulation approach is that generic expressions $\left(F_{i}\right)$ can be utilised to characterise all essential quantities associated to different aspects, $i$, of driver behaviour, e.g., speed, gas and brake activities, desired distances from obstacles and leading vehicles, stop and start performances etc. The functions $F_{i}$ take the following form:

$$
F_{i}=K_{i}(C U L T)+\alpha_{i}(C U L T) * C_{1}(A T T, E X P)+\gamma_{i}(C U L T) * C_{2}(D S)+\beta_{i}(C U L T) * C_{3}(T D) \quad \text { EQ. } 1
$$

where:

- The quantities $C_{1}, C_{2}$, and $C_{3}$ are constants that depend on driver characteristics:

$$
\begin{aligned}
& \text { if } \rightarrow A T T=1 / 8 \Rightarrow C_{1}(A T T, E X P)=1 / 4 \\
& \text { if } \rightarrow A T T=0 \Rightarrow C_{1}(A T T, E X P)=E X P \\
& C_{2}(D S)=D S \\
& C_{3}(T D)=T D
\end{aligned}
$$

- The constant $K_{i}$ and the coefficients $\alpha_{i}, b_{i}$, and $\gamma_{i}$ enable to differentiate between drivers according to their culture and, within cultures, to their personal characteristics. Their values are empirically evaluated from experimental observations and structured field tests.

The initial values assigned to the constants $C_{1}, C_{2}$ and $C_{3}$, namely $1 / 8$ and $1 / 4$ have been selected on the basis that common sense behaviour would result from the application of the proposed $F_{i}$ functions in absence of the effect of the term $K_{i}$ and the coefficients $\alpha_{i}, b_{i}$, and $\gamma_{i}$. In other words, common sense expected behaviour of a driver is obtained if the coefficients are set to the value 1 and the resulting functions $F_{i}$ are applied in SiMUD for evaluating driver behaviour. It has also been assumed that the constant $C_{1}$ is independent of experience in the case of sensation seeking, whereas it is associated to experience in the case of a prudent attitude.

In practice, the functions $F_{i}$ take different values according to the specific activity of the driver. Their values can be determined by interpolating the experimental results with the formulations assumed for each simulated activity, as will be discussed in next section.

Some examples of formulations included in the SiMUD are shown in Table II. The left side of the equations contains the quantity calculated, while the right side shows the expression containing the corresponding function $F_{i}$. The value 1.6 for the evaluation of the intended distance has been assigned based on literature studies (Treiber, Hennecke and Helbing, 2000). More complex formulations for the accelerator and brake pedals have been developed, but they are not reported in Table II for brevity.

A variety of other variables can be estimated utilising the same approach described above. As an example, the inclination of the path could have been included in the formulation considered. However, the model and simulation proposed here aim at developing simple prediction of drivervehicle interaction and therefore no other specific data have been collected for inclusion in the empirical correlations. 
Table II. Examples of formulations included in the SiMUD simulation

\begin{tabular}{|c|c|}
\hline Automotive domain formulations & Rail domain formulations \\
\hline $\mathrm{V}_{\text {intended-car }}=\mathrm{F}_{\text {car-int-speed }} \cdot \mathrm{V}_{\text {max-allowed-car }}$ & $\mathrm{V}_{\text {intended-train }}=\mathrm{F}_{\text {train-int-speed }} \cdot \mathrm{V}_{\text {max-allowed-train }}$ \\
\hline reaction_distance ${ }_{\text {car }}=\frac{\text { visibility }}{\mathrm{F}_{\text {car-react-dist }}}$ & reaction_distance $_{\text {train }}=\frac{\text { visibility }}{F_{\text {train-react-dist }}}$ \\
\hline intended_distance $_{\mathrm{car}}=\frac{1.6 * \text { speed }_{\mathrm{car}}}{\mathrm{F}_{\mathrm{car}-\mathrm{int}-\mathrm{dist}}}$ & acc $=F_{\text {train-cruise-acc }} \cdot\left(1-\frac{\text { speed }_{\text {train }}}{\text { inten_speed }}\right)$ \\
\hline
\end{tabular}

\subsubsection{Implementation of the SiMUD in the overall DVE system}

The implementation of the SiMUD within the overall DVE simulation architecture requires that the Vehicle and Environment are represented and react according to driver actions, creating a dynamically evolving system.

The vehicle simulation is associated to standard models of automotive and rail systems. The simulation of the automobile has been based on the so called "bicycle model" (Milliken and Milliken, 1995). Overall and lateral dynamics of the vehicle have been inserted in the model in parallel to the control part, governed by the driver model, in order to carry out the appropriate correction to the angle of the steering wheel, derived from the frontal and rear wheel cornering. Numerous equations have been implemented with the aim to reproduce a vehicle with six degrees of freedom. Included in the lateral dynamics of the vehicle are also the equations that govern longitudinal dynamics, making it possible to consider the lateral dynamics as an expansion of the model of the longitudinal dynamics.

The train model includes a number of differences compared to the car, as no lateral control and steering tasks are required and the only available controls are acceleration and brake levers. The forces acting on the vehicle are: a) traction, i.e., the motion force determined by the engine; $b$ ) braking, i.e., the force determined by the train device; c) rolling resistance, which is a force determined by friction on the rail, air and curve resistance; and d) curve resistance, which occurs in non rectilinear rail sections and is determined by the adaptation of the rigid structure of the train to the curvilinear path. Traction and braking forces are determined by the engine of the train and depend on the current train speed.

The simulation of the environment contains a limited amount of dynamically changing properties for both the train and car systems. Few differences occur between different types of environment. The main purpose of the environment is to provide a set of values, generally physical constants, describing the characteristics of the surrounding world. This includes gravity and air density, which are used by the vehicle model to compute forces, as well as traffic and the characteristics of other vehicles along the path. When present, traffic is the only dynamic component of the environment, since position and speed of vehicles can change over time.

Another crucial contribution of the environment is the definition of the path that the vehicle will follow. Its characteristics will vary according to the domain. For instance, in the case of automotive environment, the width of the road is essential to evaluate lateral position with respect to the middle of the carriageway, while this feature is not required by the railway description. The description of the road is provided in the open drive format, while for the railway an ad hoc 
solution has been chosen. A similar approach has been utilised for the maritime environment as discussed later.

Some examples of results of running the DVE simulation are shown hereafter, without adapting the correlations and parameters to experimental results. Figure 3 shows speed and controller responses for a simulation of a car-following task scenario (left) and a train cruise and stop task scenario (right). The driver characteristics for the car-follow scenario are: prudent, experienced, fatigued, with low task demand (Table I). The coefficients $K, \alpha, \gamma$ and $B$ functions have been set to standard values not correlated to the experiments, namely, for the gas and brake ratio all coefficients were set to 0.25 . In the case of the train cruising and stop scenario, the driver parameters are set to experienced, alert, sensation seeker and low task demand (Table I) and, for acceleration and brake, $K, \alpha, b$ and $\gamma$ were set to 10 . The values 0.25 for car-drivers and 10 for train-drivers for the coefficients $K, \alpha, \beta$ and $\gamma$ have been assigned not-correlated to experiments in order to achieve commons sense performances for the specific type of driver considered.

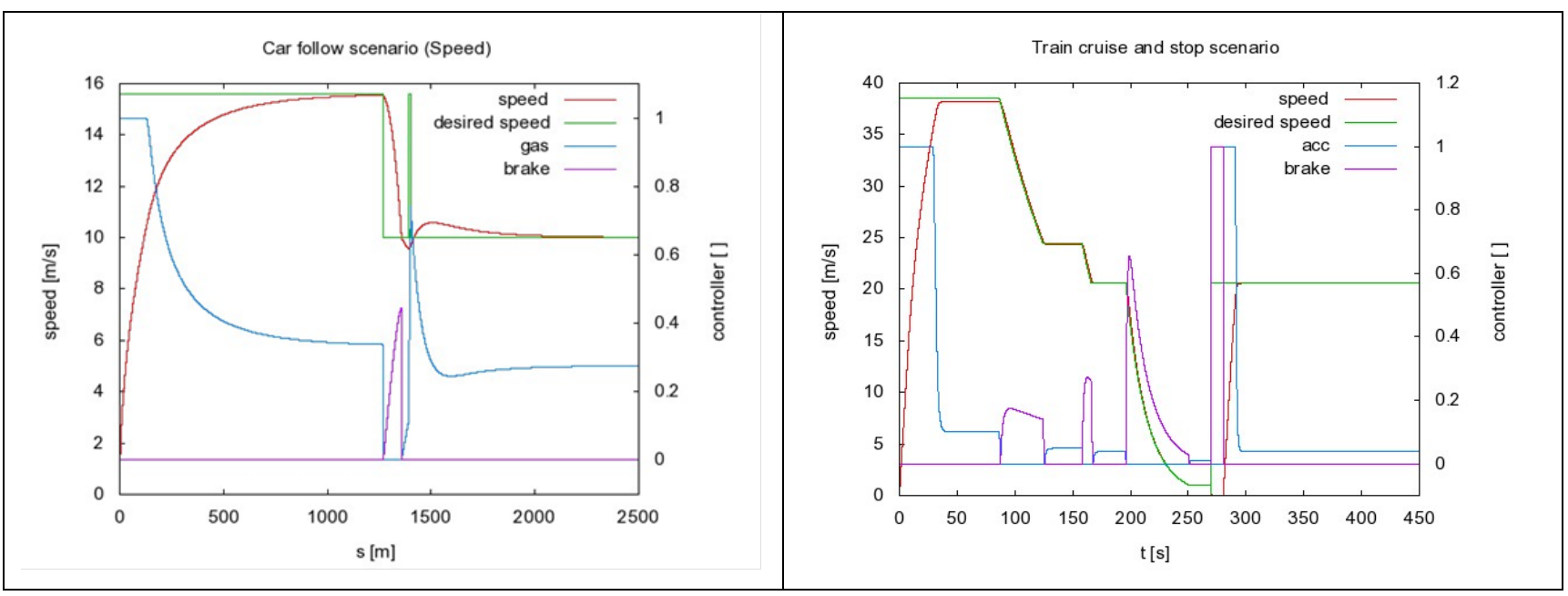

Figure 3. Speed and controllers behaviour while performing a car-following task (car scenario - left) and cruise and stop task (train scenario - right).

These results show that the SiMUD simulation is able to reproduce logical and reasonable driver behaviours, integrated in an overall DVE interaction architecture for different modes of transportation. In the following sections, tuning and validation processes are discussed. The first one has enabled adaptation of the parameters to capture differences in real observable driver behaviour and the second one has verified the ability of the simulation to reasonably predict behaviour, enabling the extension of the simulation architecture to represent also the human performance in the maritime domain.

\section{Tuning the Simulation to Automotive and Rail Domains}

To test the assumptions made in the modelling process and to estimate values of constants and coefficients of the simulation, a variety of data collected in a set of driving simulator studies were utilised. A stepwise process was adopted. Firstly, the importance of the parameters of the UMD, i.e., attitude, experience, driver state, task demand and culture, was tested. Then, the constants and coefficients of the SiMUD were estimated to allow the simulation to reproduce observed 
differences in behaviour of drivers with different sets of underlying factors controlling their behaviour.

\subsection{Driving simulator experiments to test the UMD and to tune the SiMUD}

The set of driving simulator studies used to test the UMD and to estimate constants and coefficients in the SiMUD consisted of car and train driving experiments. These experiments were conducted on a portable car/train driving simulator and in the in the full scale train operator simulator at the Swedish National Road and Transport Research Institute (VTI) and in the moving base car driving simulator at the University of Leeds (Figure 4).

Experiments with the portable driving simulator platform were carried out in Italy, Israel, France, the UK and Sweden. The same car and train driving scenarios were used in all car and train driving experiments, respectively. The use of common scenarios in a combination of a portable driving simulator platform and full scale simulators allowed simultaneous estimation of the impact of the culture factor and validation of the portable simulator platform. In total, the data set collected during the car driving simulator experiments contained data from 154 and 29 participants in the portable and moving base simulators, respectively. Among these participants there were 82 female and 101 male. Similarly, the numbers of train drivers in the data set were 78 and 32, in the portable and full scale simulators. The gender distribution of the train drivers was 9 female and 101 male. The few female train drivers in the data set are a consequence of difficulties in the recruitment of female train drivers and a reflection of the gender distribution among train drivers in general. Further details on the participants included in the data set are given by Peters et al. (2012).

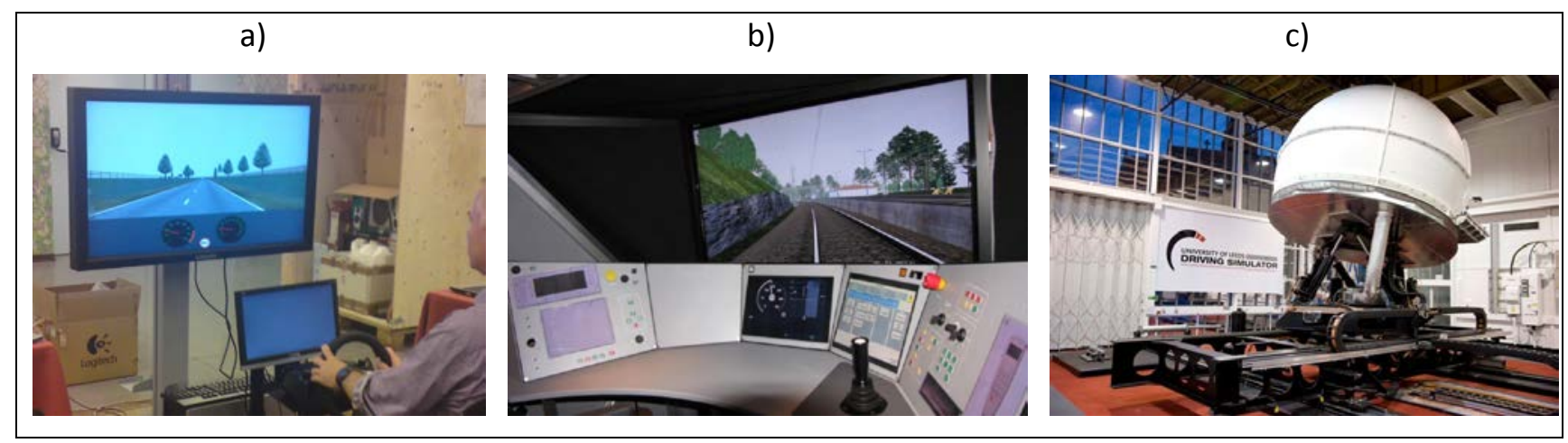

Figure 4. Driving simulators used in the experiments: a) Portable train/car driving simulator, b) Full scale train operator simulator at VTI and c) Moving base car driving simulator at the University of Leeds.

The car driving scenario consisted of two-lane highway driving assisted by an Intelligent Speed Adaptation System (ISA) and motorway driving with a Forward Collision Warning System (FCW). The ISA part of the car experiment included negotiating speed limit changes and sharp curves, as well as driving through villages and a school zone. The ISA system gave an auditory and visual warning to the driver, if the driver exceeded the speed limit with more than $2 \mathrm{~km} / \mathrm{h}$. In the FCW part of the car experiment, the driver encountered events with a lane changing truck, a road work with a lane drop, the sudden braking of a car in front and breakdown of a downstream vehicle. During these events, the FCW system issued an auditory warning if the driver came closer to the lead vehicle than a warning distance. The warning distance was based on the stopping distances of the lead and following vehicles. During the train driving scenario the drivers were asked to drive the train according to a given timetable. The train driving scenario included several changes in 
speed limit and stations at which the driver were to stop. The changes in speed limit and the stops included different track routes, i.e. slopes and levels of the permitted speeds. The driver was assisted by the a display based on the European Train Control System (ETCS) that showed the actual speed, permitted speed and issued a continuous warning (visual and auditory) if the speed limit was exceeded.

As mentioned above, culture was manipulated by the performance of identical simulator experiments in different countries. The remaining factors of the UMD, attitude, experience, driver state and task demand were considered in the driving simulator studies in the following ways. Attitude was measured using the Brief Sensation Seeking Scale (BSSS) (Hoyle et al. 2002). Experience was measured by the number of years of train driving or holding a car driving license. Driver state is in the experiment varied between participants in a dichotomous way by asking a group of "alert" participants to drive the simulator in the morning and a group of "fatigued" participants to drive the simulator after lunch dip. Lastly, Task Demand was varied by asking each participant to perform different backwards counting tasks during the experiment. More information on the driving simulator studies, including the experimental design is given by Barnard et al. (2011).

\subsection{Hypothesis Testing and Tuning Method}

The importance of the underlying factors assumed to influence driver behaviour in the UMD was analysed based on formulation and analysis of variance based testing of hypotheses regarding the drivers' behaviour during normal driving and in critical situations in which the ISA, FCW or ETCS systems issued warnings. Details of the method applied and the hypothesis testing are presented by Peters et al. (2012).

The behaviour of the driver in the SiMUD is controlled by the function $F_{i}$ specified in EQ. 1. Each function $F_{i}$ is determined by a set of coefficients and a constant. These functions enable to tune the SiMUD with respect to the observed driver behaviour and behavioural differences between drivers. For this task, linear regression analysis of the driving simulator data was performed. The method applied can be described as follows. For each function, $F_{i}$, specific parts of the driving simulator scenarios were selected. The basis for the selection was that the driving task considered had to be observable during the selected part of the scenario. As an example, in order to estimate

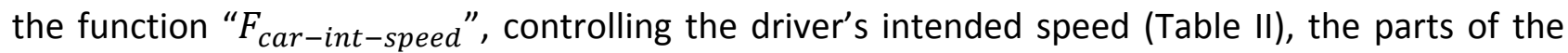
car driving scenario, in which the driver was influenced only by the speed limit, were selected. Similarly, to estimate the function controlling the intended distance, parts of the driving simulator scenario was selected in which the driver was following a vehicle for at least one minute without changing lane.

In the estimation of the function controlling the desired speed of the train drivers, sections of the railway scenario with a constant speed limit was selected. The reaction distance was estimated based on the observed distances at which the drivers responded to signals by changing the position of the accelerator or the brake lever. The regression analyses on the train data have not been fully carried out because of the similarity between the domains makes the results obtained in the car driving domain useable, at least in part, for the experiments performed in the train domain. Validation of tuning process was realized among direct comparison between the results of SiMUD and a new set of values recorded during new experiment. Further discussion on the estimation process, including the scenario selection, can be found in Hjälmdahl et al. (2012).

An inherent uncertainty in the estimation of functions controlling drivers' intentions is that only actions can be observed. In the analysis, one has to assume that the drivers are rational in the 
sense that if possible they follow their intentions. To avoid transient effects due to changes in the scenario that may influence the driver's intentions, data from the boundaries of the selected parts of the scenario were not included in the analysis. Care was also taken to ensure that all combinations of the driver parameters, i.e. ATT, EXP, DS, WL and CULT, were represented in the selected data set. Attitudes/personality (ATT) was, as described above, measured by the participants score on the BSSS. Based on the BSSS score, the participants were divided into two groups. Regarding experience (EXP), the participants were classified as experienced or novice depending on their respective number of years as licenced drivers. To ensure drivers in both alert and fatigue driver state $(D S)$, half of the group of participants drove the simulator in the morning and the other half drove after lunch, i.e., fatigue due to a post lunch dip was assumed. Workload $(W L)$ was, for all participants, manipulated during the simulator drive by assignment of secondary tasks in the form of backwards counting aloud. Finally, culture (CULT) was represented by the nationality of the participants.

\subsection{Results of the tuning process for cars}

The hypothesis testing revealed significant impacts of the culture (country) factor for almost all of the situations analysed. Task demand was also found to influence several aspects of the driving task. Attitude, Experience and Driver State was found to impact driver behaviour to a lesser extent. The constants controlling culture and the task demand coefficients were consequently expected to, in general, have a larger impact than the attitude, experience and driver state coefficients on the functions $F_{i}$ (EQ. 1.).

Examples of the constants and coefficient values obtained from the estimation process, for the car functions shown in Table II, are given in Table III. The values of the CULT parameter correspond to the countries in which experiments with the portable driving simulator platform were carried out.

Table III. Example results of the function estimation, standard errors in brackets

\begin{tabular}{|c|c|c|c|c|c|}
\hline$F_{i}$ & CULT & $K_{i}$ & $\alpha_{i}$ & $\gamma_{i}$ & $\boldsymbol{\beta}_{i}$ \\
\hline \multirow{5}{*}{$F_{\text {car-int-speed }}$} & $\mathrm{FR}$ & $0.957(0.000)$ & $0.036(0.001)$ & $-0.077(0.002)$ & $0.002(0.002)$ \\
\hline & $\mathrm{IL}$ & $0.960(0.001)$ & $0.228(0.003)$ & $-0.065(0.003)$ & $0.165(0.004)$ \\
\hline & IT & $0.892(0.001)$ & $0.104(0.003)$ & $-0.187(0.002)$ & $-0.121(0.003)$ \\
\hline & SE & $0.967(0.000)$ & $-0.105(0.001)$ & $0.047(0.001)$ & $-0.003(0.002)$ \\
\hline & UK & $0.918(0.000)$ & $0.011(0.001)$ & $0.071(0.001)$ & $-0.019(0.002)$ \\
\hline \multirow{5}{*}{$F_{\text {car-int-dist }}$} & FR & $0.831(0.002)$ & $-0.115(0.011)$ & $0.187(0.012)$ & $-0.238(0.018)$ \\
\hline & $\mathrm{IL}$ & $0.621(0.001)$ & $0.447(0.004)$ & $0.558(0.006)$ & $0.451(0.008)$ \\
\hline & IT & $0.387(0.018)$ & $0.899(0.081)$ & $-0.107(0.055)$ & $0.602(0.092)$ \\
\hline & SE & $0.695(0.001)$ & $-0.389(0.005)$ & $0.300(0.004)$ & $0.509(0.006)$ \\
\hline & UK & $0.671(0.001)$ & $-0.371(0.004)$ & $-0.101(0.005)$ & $1.120(0.010)$ \\
\hline \multirow{5}{*}{$F_{\text {car-react-dist }}$} & $\mathrm{FR}$ & $1.148(0.043)$ & $-0.082(0.226)$ & $-0.459(0.264)$ & $0.889(0.414)$ \\
\hline & IL & $1.256(0.089)$ & $0.177(0.421)$ & $-0.315(0.519)$ & $0.784(0.798)$ \\
\hline & IT & $1.208(0.175)$ & $0.516(0.777)$ & $-0.975(0.547)$ & $2.105(0.873)$ \\
\hline & SE & $1.158(0.041)$ & $-0.243(0.224)$ & $0.230(0.190)$ & $0.576(0.295)$ \\
\hline & UK & $1.240(0.062)$ & $-0.496(0.352)$ & $-0.820(0.439)$ & $1.484(0.697)$ \\
\hline
\end{tabular}

To illustrate the ability of the tuned SiMUD to reproduce observed variations between drivers with different sets of parameters, the intended speeds of experienced, prudent, fatigued drivers with low task demand are shown in Table IV together with the intended speeds of experienced, 
sensation-seeking, alert drivers with low task demand. In the computation of the intended speeds, the speed limit was set to $110 \mathrm{~km} / \mathrm{h}$.

Table IV. Intended speeds of the estimated SiMUD for two sets of driver behaviour parameters

\begin{tabular}{|c|c|c|}
\hline & $\begin{array}{c}\text { Experienced, prudent, fatigued, } \\
\text { low task demand }\end{array}$ & $\begin{array}{c}\text { Experienced, sensation-seeker, alert, } \\
\text { low task demand }\end{array}$ \\
\hline CULT & $\mathrm{v}_{\text {int-car }}[\mathrm{km} / \mathrm{h}]$ & $\mathrm{v}_{\text {int-car }}[\mathrm{km} / \mathrm{h}]$ \\
\hline $\mathrm{IL}$ & 111.9 & 115.0 \\
\hline FR & 106.8 & 107.3 \\
\hline IT & 100.4 & 101.9 \\
\hline SE & 104.2 & 102.8 \\
\hline UK & 99.9 & 100.0 \\
\hline
\end{tabular}

As can be seen in the Table IV, there is a clear effect of the culture factor. A within culture effect for the two types of drivers can also be observed. Note that the obtained intended speeds are lower than expected in real traffic, experience shows that, in real traffic many drivers tend to driver faster than the speed limit. However, they are consistent with the speeds in the driving simulator data set used for the model estimation and the aim of the model estimation was not to represent real driving but to verify the ability of SiMUD to reproduce observed differences in driver behaviour for drivers with different sets of underlying factors controlling driver behaviour.

The results of the estimation process indicate that it is possible to include common behavioural processes of drivers of different surface vehicles in a unified modelling architecture that is adaptable to specific modes of transport. The validity of this approach will be explored in the following section.

\section{Validation of the UMD in the Rail and Shipping Domains}

The validity of the SiMUD approach and its applicability to the shipping domain were explored based on data collected in an additional set of train driving and ship operator simulator experiments. These validation processes are described in this section.

\subsection{Results of the validation process for train}

To validate the results of tuning process on the train part of SiMUD, a new set of experiments were carried out in the rail driving simulator of the University of Valenciennes and HainautCambrésis on the COR\&GEST (Rail driving \& traffic monitoring in Figure 5).

Results of tuned SiMUD were compared against real drivers for final validation. Experimental setup has been designed according to driving studies used to test the UMD. 32 subjects distributed for two levels of experience, two levels of sensation seeking and gender was carried out. The drivers drove with one system, the French KVB (Speed Control by Beacons). Because the validation experiment was carried out in France, the Culture parameter was fixed to "France" value in SiMUD. Driver State was also fixed, to the level "alert", as it was decided in Peters et al. (2012) that it was too time consuming and costly to be included. For the factors Experience and Attitude, drivers were selected to include both novices and experienced and High/Low Sensation Seekers. Finally, three levels of Workload were manipulated during simulation. 
Workload was varied during the experiment by assigning a secondary task, in the form of backwards counting aloud, to the participants during the simulator drive. In the low workload condition no secondary task was assigned and in the medium and high workload conditions the participants were asked to continuously count backwards every integer and every $7^{\text {th }}$ integer, respectively.

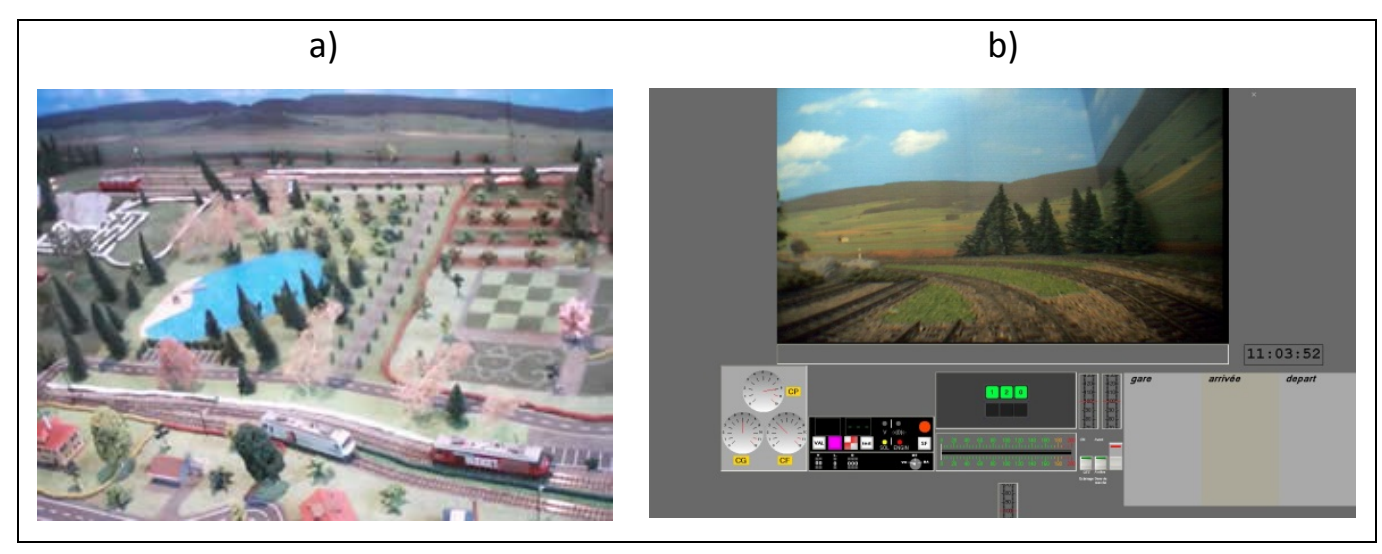

Figure 5. Driving simulator used in the validation experiments: a) COR\&GEST miniature railway platform b) Train driving interface

For each speed reduction event, the difference between measured and permitted speed behaviour indicators will be calculated at three points (PI, PII and PIII) and over the two sections connecting these three points ( $\mathrm{SI}$ and SII). PI corresponds to the start of the event, PII is defined as the point at which the driver is supposed to reduce the speed and PIII is the end point of the event (the point at which the new speed should have been reached). $\mathrm{SI}$ is the section in between points $\mathrm{PI}$ and PII. Similarly, SII is the section between points PII and PIII. For the sections, SI and SII, the performance indicator used is the mean difference between permitted and measured speed. An example of speed reduction that illustrates the points and sections is shown in Figure 6 . The zigzag speed reduction profile is due to mechanical limits of the simulator but cannot be observed by drivers when decelerating. 


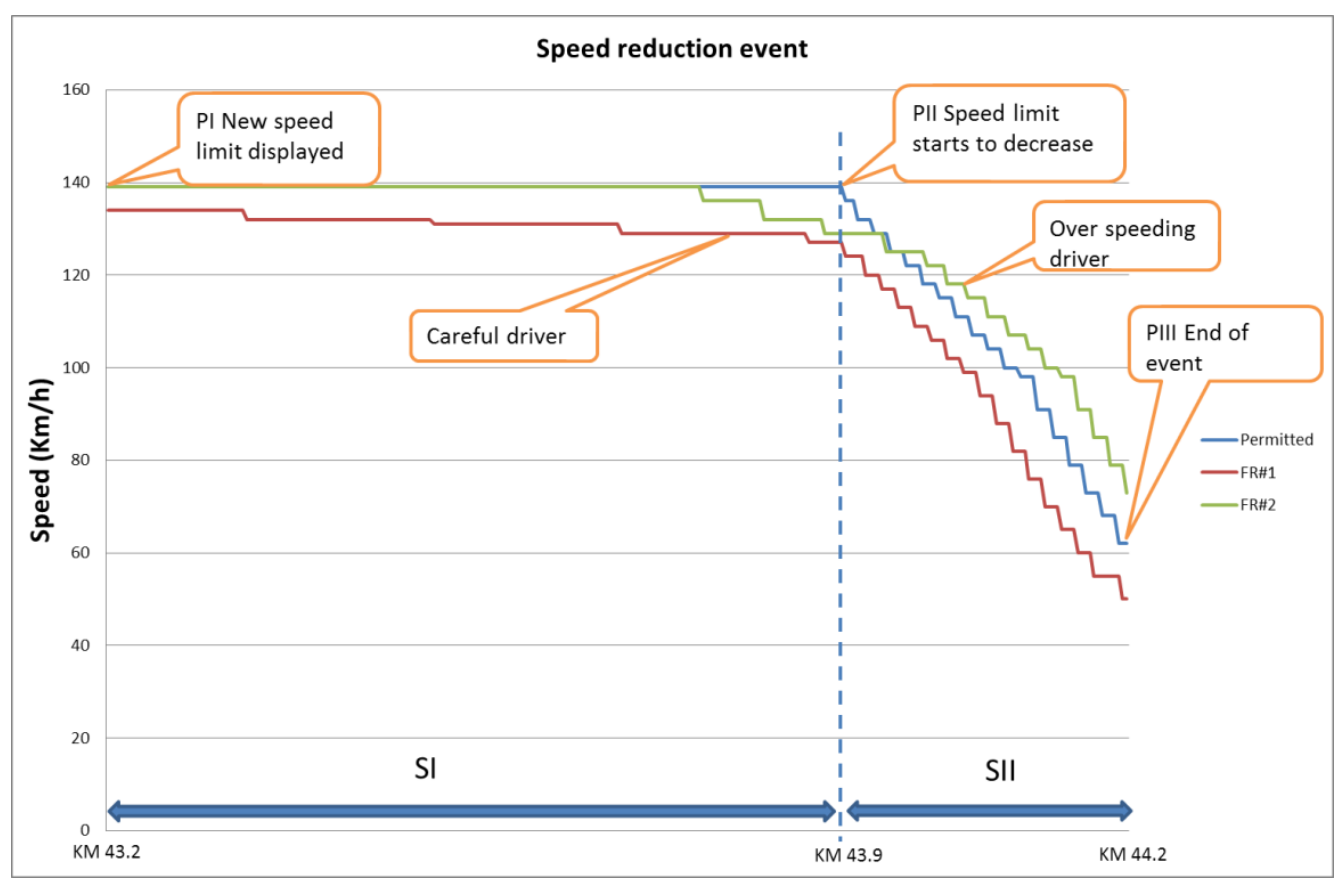

Figure 6. Example of strong speed reduction event including speed profiles for two participants.

The mean differences of speed between permitted and measured values are compared for real drivers (Table V) and SiMUD (Table VI). Same results can be observed for the speed reduction while driving events. During the first constant part of the deceleration event (section SI from PI to PII), SiMUD keeps speed few kilometres per hour under maximum permitted speed, i.e., waiting to reach PII to reduce speed, whereas real drivers anticipated deceleration. Then, during the second part (section SII from PII to PIII), SiMUD initially decelerates then recovers to new to maximum permitted speed and real drivers, that had previously anticipated deceleration, only recover few kilometres per hour under new limitation.

The SiMUD reduces speed at last moment before deceleration point without any anticipation and the braking is smoother. This effect is most likely due to the fact that the portable simulators used to tune the model did not give the same sensation of speed to drivers and so real drivers anticipated a lot and they brake harder during validation experiments.

Some specific limits on train simulation were here highlighted. If the Attitude is set to Sensation Seeker, the parameter $C_{1}$ of EQ. 1 will be equal to $1 / 4$ for any value of the experience. So the output of the $C_{1}$ function, of the $F$ function and of all the simulation does not change for: $\mathrm{ATT}=$ Sensation Seeker, EXP=Novice or EXP=Experienced. Moreover, tuning of SiMUD for experimented support system and simulator is needed for more accurate results, i.e., train model should be updated to fit better with experimental simulator and piloting is necessary to increase the confidence drivers have in the speed warning system. Despite these limits that should be easy to overcome, the general validation which consists in comparing results of speed profiles (see for example a strong speed reduction event shown in Figure 7) shows that the SiMUD manages to reproduce the behaviour of French drivers with approximately the same speed profile. 
Table V. Mean difference of speed between permitted and measured (in kilometres per hour) for the strong speed reduction events during validation experiments

\begin{tabular}{|l|l|l|c|c|c|c|c|}
\hline Experience & Sensation Seeker & Workload & PI & SI & PII & SII & PIII \\
\hline \multirow{3}{*}{ Novice } & \multirow{2}{*}{ Low sensation seeker } & Low & 2.44 & 36.58 & 72.16 & 21.63 & 3.33 \\
\cline { 3 - 7 } & & Medium & 4.22 & 31.69 & 63.17 & 18.64 & 6.66 \\
\cline { 3 - 8 } & & High & 4.55 & 33.36 & 66.50 & 21.37 & 6.44 \\
\hline \multirow{2}{*}{ Experienced } & \multirow{2}{*}{ Low sensation seeker } & Low & 5.67 & 47.11 & 78.81 & 24.34 & 8.00 \\
\cline { 3 - 8 } & & Medium & 8.50 & 48.84 & 81.00 & 25.01 & 6.66 \\
\cline { 3 - 8 } & & High & 6.83 & 46.98 & 84.16 & 25.38 & 9.67 \\
\hline \multirow{2}{*}{ High sensation seeker } & Low & 3.80 & 39.09 & 75.76 & 24.12 & 7.99 \\
\cline { 3 - 8 } & & Medium & 4.06 & 46.71 & 70.23 & 23.68 & 7.73 \\
\cline { 3 - 7 } & & High & 5.46 & 44.81 & 75.43 & 24.05 & 9.13 \\
\hline
\end{tabular}

Table VI. Mean difference of speed between permitted and measured (in kilometres per hour) for the strong speed reduction events with SiMUD

\begin{tabular}{|l|l|l|c|c|c|c|c|}
\hline Experience & Sensation Seeker & Workload & PI & SI & PII & SII & PIII \\
\hline \multirow{3}{*}{ Novice } & \multirow{2}{*}{ Low sensation seeker } & Low & 5.97 & 6.28 & 6.02 & 51.87 & 0.57 \\
\cline { 3 - 8 } & & Medium & 6.00 & 5.99 & 5.99 & 50.46 & 0.54 \\
\cline { 3 - 8 } & & High & 5.95 & 6.00 & 6.04 & 49.90 & 0.57 \\
\hline \multirow{3}{*}{ Experienced } & \multirow{2}{*}{ Low sensation seeker } & Low & 4.61 & 4.70 & 4.72 & 51.97 & 0.36 \\
\cline { 3 - 8 } & & Medium & 4.68 & 4.70 & 4.72 & 49.97 & 0.41 \\
\cline { 3 - 8 } & & High & 4.57 & 4.66 & 4.68 & 50.70 & 0.36 \\
\hline \multirow{2}{*}{ High sensation seeker } & Low & 3.95 & 4.00 & 4.07 & 49.25 & 0.24 \\
\cline { 3 - 8 } & & Medium & 2.88 & 4.08 & 4.11 & 49.79 & 0.20 \\
\cline { 3 - 8 } & & High & 4.01 & 4.02 & 4.04 & 49.47 & 0.30 \\
\hline
\end{tabular}

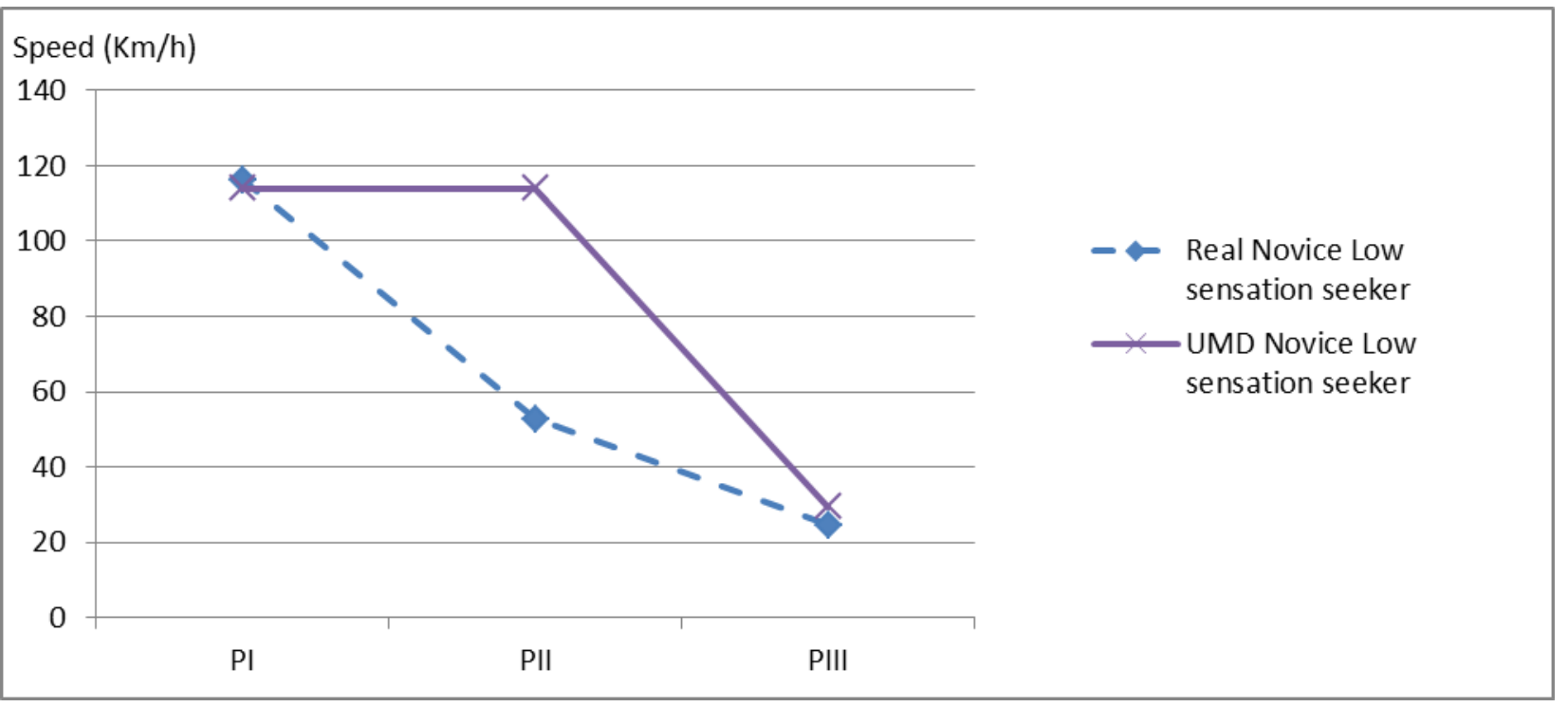

Figure 7. Example of speed (in kilometres per hour) for the strong speed reduction event $(120 \mathrm{Km} / \mathrm{h}$ to $30 \mathrm{Km} / \mathrm{h}$ ) depending on simulator (Novice \& Not Sensation Seeker). 


\subsection{Applicability of the UMD in the Shipping Domain}

The purpose of this activity was primarily to demonstrate that the generic UMD model developed and tuned in the automotive and train domains was also applicable to the shipping domain. It should be noted that manoeuvring a vessel on water is very different from road traffic and trains. However, despite the differences between the domains, similar experimental setups were developed with the aim to allow a more straightforward comparability and analysis of the results of simulator runs.

\subsubsection{Shipping Simulation}

The simulation was conducted in two full mission bridge simulators at Chalmers University of Technology in Sweden (Figure 8), were two assistance systems related to speed management and collision avoidance were tested.

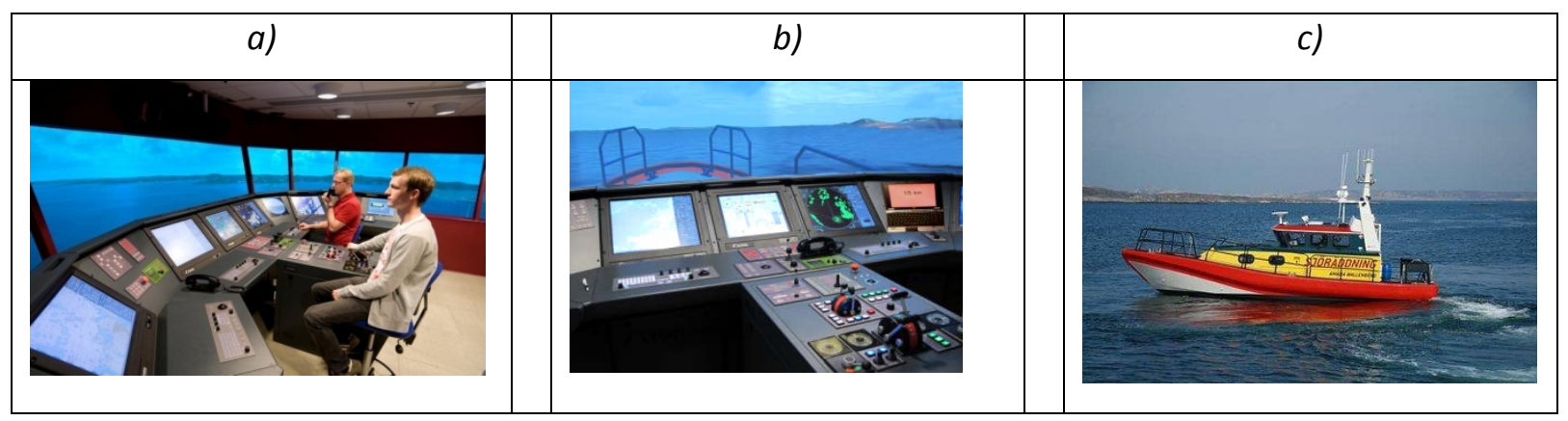

Figure 8. Full bridge simulator used in the experiment: a) View of bridge, b) Instrument panel and front view for pilot during simulation and c) Rescue service boat in real life.

The speed management system is based on geographical location and possible speed restrictions, were a warning is given for small defined areas. The collision avoidance system is based on projected closest distance to other vessels, by calculating the expected passing distance and time to that distance. The warning is provided using visual and sound displays.

In total, the data collected during the ship simulator experiment contained information from 32 participants, $50 \%$ of whom were novices and $50 \%$ experts. The participants navigated a small water jet driven vessel in high speed, in the western Swedish archipelago. It was 12 meter long boat, capable of 37 knots and highly manoeuvrable environment. Condition during the simulation was good weather, little wind, good visibility, with a limited number of other ships, with no interaction to increase the realism.

The experimental design used a $2 \times 2 \times 3$ matrix, covering 2 types of participants (novices versus experts), 2 levels of sensation seeking (low versus high) and 3 levels of workload (low, medium, and high). The type of participant and sensation seeking was a between participants design, the workload was a within participant design and developed with the Rating Scale of Mental Effort, RSME (Zijlstra, 1993). Both assistance systems were evaluated by the same experimental design (Kircher et al. 2011).

In the train and car domain half of participants performed simulation runs in fatigue condition. However, for the shipping domain, the efficacy of the fatigue induction measure was more questionable due to praxis of long working hours on a ship. Pilot runs to assess the efficacy of fatigue was performed in a pre-study, with no significant results, and therefore excluded in the experiment. Culture (nationality) was also excluded in the design, as only Swedish participants participated in the experiment. Further information can be found in Kircher et al (2011). 


\subsubsection{Hypothesis Testing and Data Collection}

Based on the UMD, the following overall hypotheses for both speed management and collision avoidance systems were defined and tested: 1) Experienced operators will receive fewer warnings than inexperienced operators; 2) Sensation seeking operators select assistance system settings which are more forgiving; and 3) Operators will receive most warnings in the low workload area.

During the experiments data were collected from the simulation for a variety of measures such as, position, speed, hydrodynamic data etc. The scenarios enabled to collect data related to workload, as the route consisted of three parts. Workload was a result of environment (low at open seas), traffic (medium due to traffic and shallow water) and adding a secondary task to traffic (high due to traffic, shallow water and counting backwards in steps of seven).

Questionnaires were used to collect data on: demographic relation, experience and life style; including the 8-item Brief Sensation Seeking Scale (BSSS) (Hoyle, et al., 2002) for capturing driver behaviour and driver attitude data; data were also collected in relation to speed management system and collision avoidance system.

The collected data was only statistically analysed when relevant for the hypothesis testing. They were carried out on IBM-SPSS, Advanced version 19.0, with a confidence interval of $95 \%$ and an alpha level of 0.05 . The alpha levels were only reported if above or below 0.05 .

\subsubsection{Results from the Validation Process}

Model validation can be understood and performed in different ways. In the car and train domain there was a process to tune and adapt the model further. This was not the case for the shipping domain, as no further experiments were performed. Consequently, as already mentioned, the current validation aims to define if the UMD model is applicable to the maritime domain.

The hypotheses associated to the issue if experienced operators will receive fewer warnings than inexperienced operators were rejected. Experience did not have any influence on the number of warnings received for both systems.

The group of hypotheses suggesting that sensation seeking operators select assistance system settings which are more forgiving resulted in differences between the two systems. For the speed management system there were no significant differences. However, for the collision avoidance system there was a statistically significant difference, which could be traced to sensation seeking operators seeking stimulation to cope with monotonous situations (Table VII).

Table VII. Numbers of warnings received for each system by the operator not following recommendations from the systems.

\begin{tabular}{|l|l|l|l|l|}
\hline \multirow{2}{*}{ Sensation Seeker } & \multicolumn{2}{|l|}{ Speed Management } & \multicolumn{2}{l|}{ Collision Avoidance } \\
\cline { 2 - 5 } & Mean & SD & Mean & SD \\
\hline $\begin{array}{l}\text { Low sensation seeker (18) } \\
\text { (Novice 6 and Expert 12) }\end{array}$ & 6.4 & 5.9 & 38.4 & 12.9 \\
\hline $\begin{array}{l}\text { High sensation seeker (14) } \\
\text { (Novice 9 and Expert 4) }\end{array}$ & 10.1 & 10.9 & 37.5 & 7.9 \\
\hline
\end{tabular}

As the number of subjects was limited, it was not possible to analyse the warning preferences for the two systems, nor the relation of novices vs. experts. However, when the simulations were 
combined with the free-text comment given by participants, it was possible to hypothesize that the two groups preferred different settings for the systems, namely:

- High sensation seekers have a tendency to seek early knowledge of situations they are likely to face by creating warning thresholds in the settings. This aims to create some sort of navigation and speed freedom.

- Low sensations seekers did not use warnings thresholds to the same extent.

At the same time, as the difference between low and high sensation seekers was relatively small the issue of using warning thresholds to cope with warnings from the two systems remain unclear.

The hypotheses proposing that operators will receive most warnings in the low workload area resulted in differences between the two systems. For the collisions avoidance system there were no significant differences. For the speed management system there was statistically significant difference, especially in the low workload area, which could be traced to operators feeling underloaded and not taking enough care of speed restrictions (Table VIII).

Table VIII. Number of warnings received for each system by the operator for the three workload levels.

\begin{tabular}{|l|l|l|c|c|}
\hline \multirow{2}{*}{ Workload } & \multicolumn{2}{|c|}{ Speed Management } & \multicolumn{2}{c|}{ Collision Avoidance } \\
\cline { 2 - 5 } & Mean & SD & Mean & SD \\
\hline Low & 3.44 & 3.30 & 13.40 & 6.60 \\
\hline Medium & 2.50 & 4.50 & 11.50 & 5.00 \\
\hline High & 2.10 & 2.70 & 13.10 & 5.80 \\
\hline
\end{tabular}

Another explanation could be that the Speed Management was new to them, as no such system exists nowadays. The Speed Management system was mainly created to be able to transfer human factors issues defined in the car domain to the shipping domain, which could explain the results.

The test results indicate that ITERATE assumptions concerning the underlying factors influencing human behaviour, developed and thoroughly tested in the car domain could be applicable to the shipping domain. Both instruments and methodology used in the experiment have demonstrated to be useful even if modification has been made to the used questionnaires. Especially the questionnaires for driver behaviour and driver attitude have passed through extensive modification from the original version for car drivers.

This is natural as there a number of differences between the domains; the on-board culture steering is not precise, no speed limit in most open waters, less precise regulations than in the car domain etc.. However, to our knowledge this is the first time that questionnaires and other tools from the car domain have been utilized for the shipping domain. Additional research will be needed to evaluate the appropriateness of these modifications and create a tuning process like the one used for the car domain.

\section{Conclusions}

The results of several numerical simulation runs and the comparison with experimental data and simulation observations discussed in this paper have demonstrated that the proposed approach of 
an Unified Model of Driver behaviour (UMD) is appropriate and able to account for general and common aspect of cognition and behaviour of different surface transport modes.

The implementation of the UMD into a computerised numerical simulation, called SiMUD (Simulation of Model of Universal Driver), has required an accurate work of testing, tuning and adaptation of the set of critical UMD parameters, exploiting experimental results carried out in coordinated test beds and simulator environments. This activity was primarily carried out for automotive and rail domain and the coefficients were then extrapolated to the maritime environment.

An important issue of the UMD model is the completeness of the parameter set (ATT, EXP, DS, WL, CULT). However, a discussion of this nature is out of the scope of this paper. In principle, the same methodological approach utilised for the simulation development (SIMUD) could be implemented for other possible parameterisations. This would improve the accuracy and scope of the predictive approach. As an example, likely parameters that would increase the completeness of the approach could be gender, as suggested by the driving simulator experiments.

Another important aspect is the sensitivity of the SIMUD to the parameters. The results of the predictive simulation runs illustrate the sensitivity of the model to differences in driver characteristics. The sizes of the obtained differences are in line with the differences observed in the driving simulator data. A set of predictive runs of SiMUD were compared with the real performances of "drivers" in real training simulators. The overall results of these validation tests can be summarised as follows:

1. The UMD concept represents a valuable and feasible approach, as it enables representation of the fundamental characteristics and parameters affecting human behaviour in different surface transport domains.

2. The numerical simulation approach based on the theoretical model seems able to reproduce, with a sufficiently accurate precision, the behaviour of different types of "drivers". This conclusion is valid for both rail and automotive transports, even if some adaptation is necessary in order to account for the environmental aspects of each specific domain.

3. The adaptation of the SiMUD to the maritime domain requires some more research work and possibly further experimental analysis and tuning, even if the fundamental aspects of behaviour can been captured by the current state of the simulation.

It can be concluded that it is reasonable to consider the SiMUD approach capable of describing, in a predictive way, the behaviour of drivers of different transportation modes. Consequently, it can be utilised for prospective studies associated to safety assessment and design of existing as well as new technologies. Moreover, it can be implemented in studies involving the evaluation of human performances when a driver is the ultimate decision maker in control process loops and in managing highly automated systems.

The SiMUD approach of using parameterised functions to describe drivers having different sets of underlying factors controlling behaviour could also be used together with driver models included in current traffic micro-simulation models. This would allow estimation of impacts on the traffic system level of differences in driver behaviour and interaction with support systems. Exploration of this potential is an interesting topic for further research. 


\section{Acknowledgements}

The research leading to these results has received funding from the European Commission Seventh Framework Programme (FP7/2007-2013) under grant agreement $n^{\circ} 218496$ Project ITERATE. The authors are very grateful to all the colleagues of the Project for their support and collaboration.

The authors are in debts to all the members of the ITERATE team for their continuous and fruitful support and collaboration offered during the entire duration of the Project.

\section{References}

Barnard, Y., Carsten, O. \& Lai, F. (2011). From theoretical model to experimental data: A structured approach to design experiments to seed a model of vehicle operation with new systems. In Cacciabue, P.C., Hjälmdahl, M, Luedtke, A. \& Riccioli, C., (Eds.), Human Modelling in Assisted Transportation, Springer-Verlag, 109-117.

Carsten, O. (2007). From driver models to modelling the driver: what do we really need to know about the driver? In Cacciabue, P.C. (Ed.), Modelling Driver Behaviour in Automotive Environments, Springer, London, pp. 105-120.

Hjälmdahl, M., Amantini, A. and Cacciabue, P.C. (2012). Implementation of a unified model of driver into numerical algorithms for a predictive simulation of behaviour in different transportation contexts. In de Waard, D. Merat, N., Jamson, A.H., Bernard, Y., and Carsten, O.M.J. (Eds), Human Factors of Systems and Technology. Shaker Publishing, Maastrich, the Netherlands, 359-372.

Hoyle, R.H., Stephenson, M.T., Palmgreen, P., Lorch, E.P., \& Donohew, R.L. (2002). Reliability and validity of a brief measure of sensation seeking. Personality and Individual Differences, 32, $401-414$.

Oppenheim, I., \& Shinar, D. (2012). A context-sensitive model of driving behaviour and its implications for in-vehicle safety systems. Cogn Tech Work., 13(4), 229-241.

Kircher, A., van Westrenen, F., Söderberg, H., \& Lützhöft, M. (2011). Behaviour of deck officers with new assistance systems in the maritime domain. In de Waard, D. Merat, N., Jamson, A.H., Bernard, Y., and Carsten, O.M.J. (Eds), Human Factors of Systems and Technology. Shaker Publishing, Maastrich, the Netherlands, 189-199.

Kirwan, B., \& Ainsworth, L. K. (1992). Guide to task analysis. Taylor and Francis, London.

Michon, J. A. (1985). A critical review of driver behaviour models: What do we know? what should we do? In L.A Evans and R.C. Schwing (Eds.) Human Behaviour and Traffic Safety. New York: Plenum Press, 487-525.

Milliken, W. F. \& Milliken, D. L. (1995). Race car vehicle dynamics. Warrendale, Pa. Society of Automotive Engineers. ISBN 978-1-56091-526-3.

Neisser, U. (1967). Cognitive Psychology. Appleton-Century-Crofts, New York.

Peters, B., Vadeby, A., Forsman, Å \& Tapani, A. (2012). Developing a unified model of driving behaviour for cars and trains. In de Waard, D. Merat, N., Jamson, A.H., Bernard, Y., and Carsten, O.M.J. (Eds), Human Factors of Systems and Technology. Shaker Publishing, Maastrich, the Netherlands, 343-357.

Treiber, M., Hennecke, A., \& Helbing, D. (2000). Congested traffic states in empirical observations and microscopic simulations. Physical Review, 62 (2), 185-1824

Zijlstra, F.R.H. (1993). Efficiency in Work Behavior. A Design Approach for Modern Tools. Ph.D. Thesis. Delft University of Technology, Delft, the Netherlands. 